Motrivivência $\quad$ v. $27, \quad$ n. $44, \quad$ p. $49-63$, maio/2015

\title{
DESENVOLVIMENTO DE UM MÉTODO PARA AS PESQUISAS EM POLÍTICAS PÚBLICAS DE ESPORTE NO BRASIL: uma abordagem de pesquisa mista
}

\author{
Fernando Marinho Mezzadri \\ Marcelo Moraes e Silva² \\ Katiuscia Mello Figuerôa ${ }^{3}$
}

\section{RESUMO}

O presente artigo expõe um método de pesquisa misto para o estudo das políticas públicas de esporte no Brasil. Para atender a este fim, o texto é divido em dois momentos. No primeiro, apresenta-se um modelo conceitual para a análise qualitativa das políticas públicas brasileiras direcionadas ao esporte. Esta fase também é dividida em outras duas, sendo que uma delas versa sobre a construção de um referencial teórico para a interpretação do estudo sobre as políticas públicas; e a outra demonstra um aporte conceitual metodológico baseado na sociologia de Pierre Bourdieu. Na segunda fase, desenvolve-se uma análise da parte quantitativa que visa à interpretação dos dados coletados na parte empírica da pesquisa. Feitas essas etapas será possível numa fase posterior produzir um modelo de avaliação, monitoramento e, principalmente, aprimoramento das políticas públicas para o esporte brasileiro.

Palavras-chave: Políticas Públicas de Esporte; Estado; Método de Pesquisa Mista

1 Doutor em Educação Física. Docente do Departamento de Educação Física e do Programa de Pós-Graduação em Educação Física / UFPR. Curitiba / Paraná, Brasil.

E-mail: fmezzadri@uol.com.br

2 Pós-Doutor em Educação Física. Docente do Departamento de Educação Física e do Programa de Pós-Graduação em Educação Física / UFPR, Curitiba / Paraná, Brasil.

E-mail: moraes_marc@yahoo.com.br

3 Pós-Doutoranda em Educação Física. Curitiba / Paraná, Brasil.

E-mail: ktmello@gmail.com 


\section{INTRODUÇÃO}

O interesse em estudar políticas públicas direcionadas ao esporte é algo relativamente novo no Brasil. Tal tendência inicia-se na década de 1980, justamente no período em que se inicia uma abertura democrática no país (STAREPRAVO, 2011). Segundo Mezzadri (2011), essa tendência aumentou consideravelmente após o surgimento do Ministério do Esporte, da Rede CEDES (Centro de Desenvolvimento do Esporte Recreativo e do Lazer), bem como da eleição do Brasil para sediar a Copa do Mundo de Futebol em 2014 e as Olimpíadas do Rio de Janeiro em 2016. Tais fatos atraíram olhares de vários setores do campo acadêmico e da sociedade civil organizada.

Se a produção brasileira sobre as políticas públicas de esporte ainda é algo recente (MANHÃES, 1986; LINHALES; PEREIRA FILHO, 1999; AMARAL; PEREIRA, 2009; HÚNGARO et. al. 2009; MEZZADRI, 2011; STAREPRAVO, 2011), no contexto internacional existe uma produção acadêmica mais consistente que versa sobre os sistemas de esporte e as políticas de diferentes nações (BUGGEL, 1986; CHALIP, 1995; COAKLEY, 2010; GREEN; COLLINS, 2008; GREEN; HOULIHAN, 2005; RIORDAN, 1989, 1991; SEDLACEK et. al. 1994; SEMOTIUK, 1990). Nesse sentido, o presente artigo pretende seguir as trilhas dos estudos internacionais e apresentar todo um aporte metodológico para a realização de pesquisas que qualifiquem este campo de investigação.

Dentro desta proposta de trabalho, o presente texto é composto por duas fases distintas, porém complementares e sequenciadas, a que se pode chamar de abordagem mista, visto que conecta tanto uma abordagem qualitativa como uma quantitativa no desenvolvimento de uma pesquisa (CRESWELL; PLANO CLARK, 2007; 2013; DE BOSSCHER, et. al. 2008).

Para alcançar tal intento, a primeira fase visa desenvolver um modelo conceitual para a análise qualitativa das políticas públicas brasileiras direcionadas ao esporte (MEZZADRI, 2011; STAREPRAVO, 2011; CANAN, et. al. 2014). Este momento é dividido em outras duas etapas. Na primeira, apresenta-se a construção de um referencial teórico para a interpretação do estudo sobre as políticas públicas; no segundo, é exposto o aporte conceitual metodológico baseado na sociologia de Pierre Bourdieu (1983a; 1983b; 1998a; 1998b; 2014). Na segunda, por sua vez, demonstra-se uma análise da parte quantitativa que busca a interpretação dos dados coletados na parte empírica da pesquisa. Feitas essas etapas será possível numa fase posterior produzir um modelo de avaliação e monitoramento das políticas públicas para o esporte brasileiro.

\section{Parte 1 - Bases Qualitativas}

Políticas Públicas: delimitando conceitos

De acordo com Frey (2000) e Souza (2006), o denominado "policy science" surge enquanto área de conhecimento, nos Estados Unidos na década de 1950. Os autores ainda lembram que, na Europa, esta temática entra em pauta com mais intensidade somente na década de 1970 e, no Brasil, apenas em meados dos anos de 1980. Starepravo (2011) salienta que a década de 1980 foi o período de instalação da democracia no país, o que contribuiu para alguns progressos nos moldes de gestão pública, controle e participação social, 
como também o surgimento dos primeiros estudos científicos sobre políticas públicas.

No Brasil, o termo "política" possui uma terminologia abrangente, constituído pelas instituições (partidos políticos e órgãos governamentais), pelos agentes (políticos e gestores) e pelo desenvolvimento das ações (programa e projetos), ou seja, engloba todas as dimensões possíveis do ato de governar.

Todavia, Frey (2000), ao delimitar o termo em questão no campo da ciência política, apresenta uma divisão em três partes complementares entre si: "polity", "politics" e "policy". A expressão "polity" denomina as instituições políticas, "politics" se refere os processos políticos e, por fim, "policy" estabelece os conteúdos da política. Mais especificamente, pode-se definir tais terminologias como:

- a dimensão institucional 'polity' se refere à ordem do sistema político, delineada pelo sistema jurídico, e à estrutura institucional do sistema político-administrativo;

- no quadro da dimensão processual 'politics' tem-se em vista o processo político, freqüentemente de caráter conflituoso, no que diz respeito à imposição de objetivos, aos conteúdos e às decisões de distribuição;

- a dimensão material 'policy' refere-se aos conteúdos concretos, isto é, à configuração dos programas políticos, aos problemas técnicos e ao conteúdo material das decisões políticas (FREY, 2000, p. 216-217).
Como se pode observar, o termo "política" deve ser compreendido de forma abrangente e subdividido entre as instituições (polity), os agentes (politics) e os programas (policy). Isto é, embora cada um desses elementos se constitua separadamente, com uma lógica própria, percebe-se uma articulação entre eles.

Segundo Frey (2000), o conjunto das relações entre o tripé "polity", "politics" e "policy", constitui os elementos da "policy analysis". Tais questões aproximam-se da perspectiva de Dye (1976), sobre o estudo de políticas públicas, ao defender que esse campo se refere à busca pelo entendimento sobre o que os governos fazem, porque o fazem e, principalmente, que diferença isto faz no seio de uma sociedade civil organizada. Na esteira destas definições, Ham e Hill (1993) enfatizam que um aumento do conhecimento sobre o processo de elaboração das ações de governo pode proporcionar a melhoria da qualidade das políticas públicas desenvolvidas, já que permite a extensão e a melhoria das informações disponibilizadas aos "policy makers".

Também se deve levar em consideração que as relações entre as três dimensões (polity, politics e policy) apresentam suas disputas de poder no campo político ${ }^{4}$. Mezzadri (2011) lembra que as disputas e interações entre os agentes e as instituições no interior deste campo sempre deixarão suas marcas nos programas e projetos desenvolvidos pelos governantes.

Para compreender todo o campo que envolve a política pública, torna-se

4 Neste ponto é que a aproximação com as contribuições do sociólogo francês Pierre Bourdieu, que serão exploradas no próximo tópico, se tornam profícuas e pertinentes. 
necessário avançar a discussão sobre a "policy analysis". Nesse ponto, conforme aponta Frey (2000), destacam-se duas dimensões: "policy arena" e "policy cycle". A primeira insere em seu contexto os conteúdos, os agentes e as instituições, referindo-se aos processos de conflito e consenso dentro das diversas áreas da política. As áreas da política podem ser apontadas de acordo com o seu caráter e caracterizadas quanto à forma, aos efeitos de implementação aplicados aos conteúdos das políticas e ao modo da resolução de conflitos políticos. Em contrapartida, a "policy arena", segundo aponta Souza (2006) se manifesta nas formas de apoio e de rejeição e principalmente das disputas em torno das decisões por quais passam as políticas públicas.

Já a "policy cycle" determina suas fases, desde o processo de definição das demandas sociais até a avaliação das mesmas (FREY, 2000). O ciclo completo da política pública é constituído dos seguintes estágios: a) percepção e definição do problema; b) elaboração de programas e decisão (planejamento); c) implementação de políticas; d) avaliação de políticas e correções das ações (SOUZA, 2006). A compreensão de cada uma das fases da "policy cycle" é de fundamental importância para que as políticas públicas (policy) possam ser construídas e implementadas com o objetivo de responder às demandas sociais e melhorar as condições da sociedade.

Entretanto, conforme apontam Mezzadri (2011) e Canan et. al. (2014), essa interação entre agentes e instituições na "policy cycle" depende da estratégia utilizada no processo da construção das políticas públicas. Deubel (2006) argumenta que o sentido de uma implementação vai depender da dinâmica das interações e disputas no interior da gestão pública, da participação da sociedade e das outras instituições envolvidas na área. $O$ autor ainda lembra que é de primordial importância a interpretação e a reinterpretação dos fatos ocorridos na "policy cycle" para aprimorar as ações realizadas.

\section{REFERENCIAL TEÓRICO-METODOLÓGICO}

Para a realização de uma análise teórica-metodológica mais aprofundada na área das políticas públicas, propõe-se neste momento a utilização da teoria dos campos de Pierre Bourdieu. De acordo com os trabalhos de Mezzadri (2011), Martines (2009) e Starepravo (2011) e Canan et. al. (2014), a interseção das categorias de campo político e campo esportivo, levantadas por Bourdieu, acaba por constituir o denominado subcampo das políticas públicas para o esporte. Segundo Bourdieu (1983a; 1983b; 2009; 2014), a teoria dos campos é estruturada por um processo de disputas e interações entre os agentes e/ou instituições presentes em cada campo:

Os campos se apresentam à apreensão sincrônica como espaços estruturados de posições (ou de postos) cujas propriedades dependem das posições nestes espaços, podendo ser analisadas independentemente das características de seus ocupantes (em partes determinadas por elas). (...)A estrutura do campo é um estado de relações de força entre os agentes ou instituições engajadas na luta ou, se preferirmos, da distribuição do capital específico que acumulado no curso das lutas anteriores, orienta as estratégias ulteriores. Esta estrutura, que está na origem das estratégias destinadas a transformá-la, também está sempre em jogo: as lutas cujo espaço é o campo têm por objetivo o monopólio 
da violência legítima (autoridade específica) que é característica do campo considerável, isto é em definitivo, a conservação ou a subversão da estrutura da distribuição do capital específico (BOURDIEU,1983b p. 123).

As disputas entre os agentes no interior do campo dependem dos seus habitus e dos seus respectivos capitais, sejam eles simbólicos, econômicos, sociais, políticos, físicos, culturais e/ou esportivos. Mezzadri (2011), ao aproximar a discussão com a categoria de política pública, observou que a denominada "policy arena", apresentada no tópico anterior, encontra-se próxima à teoria dos campos, pois as disputas existentes no interior da politics (instituições) e polity (agentes) são determinantes na composição e definições das políticas públicas (policy). $\mathrm{O}$ autor, ao considerar que as disputas e interações ocorrem no interior do campo, levanta as seguintes perguntas: Quem são os agentes e quais instituições estão envolvidas no desenvolvimento do esporte? Quem constrói as políticas públicas para o esporte? Como são estruturados os projetos, programas e ações de esporte? Estes três grandes questionamentos estão intrinsecamente inseridos na teoria dos campos e na composição da denominada "policy arena".

Quanto à teoria dos campos, o campo político pode ser compreendido como um lugar de concorrência entre os agentes que nele se acham envolvido e com espaços definidos por sua preferência e/ou posição (BOURDIEU, 2009; 2014). Sob essa lógica, a grande maioria dos agentes acaba ficando à margem do processo de decisão política, tendo que se contentar com o que é disponibilizado pelas instituições. Isso faz com que o campo político se apresente enquanto um dos espaços sociais mais restritivos à entrada de novos agentes. As posições são conservadas e a produção concentrada, fazendo com que o interesse da sociedade seja reduzido e interpretado a partir dos interesses dos agentes políticos.

O campo político, entendido ao mesmo tempo como campo de forças e como campo das lutas que têm em vista transformar a relação de força que confere a este campo a sua estrutura em dado momento, não é um império: os efeitos das necessidades externas fazem-se sentir nele por intermédio sobretudo da relação que os mandantes, em consequência da sua distância diferencial em relação aos instrumentos de produção política, mantêm com seus mandatários e da relação que estes últimos, em consequência das suas atitudes, mantém com as suas organizações. O que faz com que a vida política possa ser descrita na lógica da oferta e da procura é a desigual distribuição dos instrumentos de produção de uma representação do mundo social explicitamente formulada: o campo político é o lugar em que se geram, na concorrência entre o agentes que nele se acham envolvidos, produtos políticos, problemas, programas, análises, comentários, conceitos, acontecimentos entre os quais os cidadãos comuns, reduzidos ao estatuto de "consumidores", devem escolher, com probabilidades de mal entendido tanto maiores quanto mais afastados estão do lugar de produção" (BOURDIEU, 2009, p. 164).

Bourdieu (2009; 2014) ainda salienta que a entrada neste campo como agente politicamente ativo está relacionada à sua concentração de capital. Entende-se aqui capital como o conjunto de recursos atuais ou potenciais pertencentes aos agentes, que pode assumir diferentes formas: cultural, econômico, social e/ou esportivo. Dentre as formas de capital, destacam-se o capital simbólico e social como os principais 
responsáveis pela manutenção das posições dentro do campo político. Tais elementos podem ser visualizados na seguinte passagem do autor:

O Político avisado é o que consegue dominar praticamente o sentido objectivo e o efeito social das suas tomadas de posição graças ao domínio que ele possui do espaço de tomadas de posição actuais e, sobretudo, potenciais ou, melhor, do princípio dessas tomadas de posição a saber, o espaço das posições objectivas no campo e das atitudes dos seus ocupantes: este "sentido prático" das tomadas de posição possíveis e impossíveis, prováveis e improváveis para os diferentes ocupantes das diferentes posições é o que lhe permite "escolher" as tomadas de posição convenientes e convencionadas, e evitar as tomadas de posição comprometedoras que fariam com que se encontrasse com os ocupantes de posições opostas no espaço do campo político (BOURDIEU, 2009, p. 172).

Como se pode observar, o campo político é um dos campos mais fechados que existe. Suas disputas e interações ocorrem sempre entre os agentes possuidores de um capital específico. Por isso, a entrada de outros agentes no campo político torna-se cada vez mais complexa.

Tais questões também se materializam no campo esportivo. O próprio Bourdieu (1983b) explora em um dos seus diversos artigos a estrutura do campo esportivo. Para o autor, este campo representa um objeto definido e com peculiaridades próprias. Por isso, pode-se estruturá-lo em disputas que se dão nos aspectos sociais, políticos, culturais e econômicos dos agentes e das instituições esportivas.

Mezzadri (2011) amparado em tais definições de Bourdieu, salienta que as disputas e interações dentro do campo são evidenciadas pelo poder dos agentes e das instituições que são determinantes na construção das políticas públicas para o esporte. O autor compreende o campo esportivo como algo com lógica própria, ou seja, um lugar de lutas e interações entre dominados e dominantes, que disputam o monopólio do poder e melhores colocações dentro do campo. Essas disputas são encontradas nas esferas do amadorismo contra o profissionalismo, do esporte de participação contra o esporte espetáculo; do conhecimento científico do esporte contra o senso comum; dos projetos sociais de esporte contra o esporte de rendimento; das ofertas do poder público para o esporte contra a demanda social e dos praticantes, entre outras possibilidades.

Dessa maneira, a oferta da política pública e a demanda social estão cada vez mais presentes na agenda do atual campo esportivo, ainda mais ao se considerar os megaeventos esportivos que irão acontecer no país (MEZZADRI, 2011). Nessa linha de raciocínio, o consumo da produção esportiva e o capital simbólico, social e político dos agentes são determinantes na estruturação desse campo, assim como afirma Bourdieu (1983b, p.148):

Temos aqui um encontro entre a oferta, isto é a forma particular que reveste a prática, e o consumo esportivo propostos a um dado momento de tempo, e a demanda, isto é as expectativas, os interesses e os valores dos praticantes potenciais. Sendo a evolução das práticas e dos consumos reais o resultado da confrontação e do ajustamento permanente entre um e outro. É óbvio que cada momento, cada recém-chegado deve contar com um estado determinado das práticas e consumos esportivos e de sua distribuição entre as classes sociais, estado que não the compete 
modificar e que é o resultado de toda a história anterior da concorrência entre os agentes e as instituições engajadas no campo esportivo.

Como visto nas linhas acima o autor marca muito bem as relações presentes no campo esportivo. Contudo, é somente na articulação entre o campo esportivo e político que delimita-se uma interseção que é representada pelas políticas públicas do esporte (MEZZADRI, 2011; STAREPRAVO, 2011; GODOY, 2013). Essa interseção é identificada pela composição do subcampo das políticas públicas para o Esporte e construídas nas disposições das instituições, agentes, ofertas e demandas sociais. As aproximações entre a definição de políticas públicas e o modelo de análise inserido na teoria dos campos, deveriam constituir-se como elementos importantes nas ações governamentais e na base do desenvolvimento das pesquisas científicas.

\section{Parte 2 - bases quantitativas}

Construindo métodos e instrumentos de pesquisa

Henry et al. (2005), ao analisar o contexto internacional, observou que existe uma necessidade de padronização nos métodos de investigação, utilizados para fazer estudos sobre políticas de esporte. Os autores lembram que, na mesma proporção, existe uma limitação de estudos com dados disponíveis e quantificáveis sobre as políticas públicas de esporte. Se isso ocorre no cenário internacional, tais fatores são ainda mais contundentes no cenário brasileiro, conforme pode ser visualizado nos estudos de Linhales e Pereira Filho (1999), Amaral e Pereira (2009), Húngaro et. al. (2009) e Starepravo (2011).

Apesar da pouca quantidade, como aponta Henry e colaboradores, tem se visto um desenvolvimento considerável, desde 2000, de pesquisas sobre esportes e políticas públicas, principalmente por conta do desejo dos pesquisadores em explicar e compreender as causas de sucesso e/ou fracasso de alguns países em competições esportivas internacionais (OAKLEY, GREEN 2001; DIGEL, BURK, FAHRNER, 2006; GREEN, HOULIHAN, 2005; DE BOSSCHER, et. al. 2008; HOULIHAN, GREEN, 2008; BERGSGARD et.al. 2007).

Nesse sentido, se apresenta neste momento um aporte teórico-metodológico sobre políticas públicas de esporte, que articule questões teóricas elaboradas anteriormente com instrumentos de pesquisa quantificáveis. Com a elaboração e construção desse método, poder-se-á ter bases mais seguras e consistentes para uma avaliação e monitoramento adequado das políticas públicas para o esporte no Brasil.

Tomaram-se por base os estudos de Creswell e Plano Clark (2007; 2013) e De Bosscher et. al. (2008) para propor um modelo de pesquisa, que é composto por três fases. Considera-se que a primeira fase do estudo, descrita nos dois tópicos anteriores, apontava para o desenvolvimento do modelo conceitual (etapa qualitativa). Então, apresenta-se a partir deste momento a segunda etapa da pesquisa científica, baseada num método quantitativo, que busca encontrar os fatores determinantes das políticas públicas do esporte no Brasil. Durante essa fase, uma triangulação de métodos deverá ser utilizada, buscando configurar um instrumento de pesquisa para a definição dos indicadores. A terceira 
fase da pesquisa (análise) deve estabelecer novos indicadores para futuras pesquisas, no sentido de confirmar o método e, assim, aprimorar a compreensão das políticas esportivas brasileiras.

A definição do método e do instrumento da pesquisa em muitos casos é considerada o momento mais problemático de uma pesquisa, principalmente porque o esporte é incorporado em um contexto cultural mais amplo, onde as crenças, normas e valores têm um impacto significativo sobre o caráter de políticas públicas para o esporte (HOULIHAN, GREEN, 2008).

Consequentemente, os instrumentos de política são muitas vezes dependentes do regime político de cada nação, o que implica o fato de que as ações políticas semelhantes podem ter resultados diferentes em cada país ou em cada situação. Houlihan e Green (2008) argumentam que alguns setores, como o esportivo, podem ser menos enraizados na cultura de um sistema político do que outros serviços básicos. O que se pode tomar de lição das advertências do autor é que um modelo metodológico próprio deve ser construído para que as pesquisas sobre as políticas públicas de esporte no Brasil possam ser mais qualificadas, bem como apresentar resultados mais refinados.

O próximo passo, visando ao aprimoramento e refinamento destes instrumentos de pesquisa, foi a elaboração de indicadores para compor o instrumento metodológico, a fim de almejar a subsequente análise dos dados coletados. A aplicação do modelo em um ambiente empírico é necessária para entender como a política pública de esporte é ativada no Brasil e como os diferentes fatores podem ser operacionalizados em termos metodológicos.
Sendo assim, procurou-se desenvolver um método para medir em termos quantitativos os determinantes da política pública de esporte. Tais pontos visam operacionalizar o quadro conceitual num ambiente empírico estável e consistente. Durante essa fase, a já mencionada triangulação dos métodos foi utilizada (CRESWELL, PLANO CLARK, 2007; 2013 DE BOSSCHER et. al. 2008).

\section{Seleção da amostra}

A amostra da pesquisa em políticas públicas para o esporte (policy) deve ser delimitada a partir de recortes temáticos das ações, programas e projetos que os governos federal, estaduais ou municipais executam. Nessa perspectiva, utiliza-se como exemplo a proposta do governo federal que segue a Política Nacional de Esporte e os projetos desenvolvidos pelo Ministério do Esporte.

Os programas e projetos do Governo Federal estão inseridos nas respectivas estruturas organizacionais: Secretaria Nacional de Esporte de Alto Rendimento (SNEAR); Secretaria Nacional de Esporte, Educação, Lazer e Inclusão Social (SNELIS); Secretaria Nacional de Futebol e Defesa do Torcedor (SNFDT); e Secretaria Executiva.

Atualmente, os programas do Ministério estão sendo realizados da seguinte forma: SNEAR - Bolsa Atleta, Rede Nacional de Treinamento, Rede Cenesp, Centro de Iniciação ao Esporte, Plano Brasil Medalhas, Brasil Potência Olímpica, Jogos Militares, Jogos Escolares e Rio 2016; SNELIS - Programa Esporte Lazer nas Cidades - PELC, PELC/ Vida Saudável, Rede CEDES, Programa Segunda Tempo, Atletas na Escola.

Após a coleta de dados das ações, programas e projetos esportivos realizados 
pelo Governo Federal, inicia-se a segunda etapa da seleção da amostra, que versa sobre as atuações realizadas pelos outros entes federativos, a saber, governos estaduais e prefeituras municipais.

\section{Coleta de Dados}

A coleta de dados deve ser baseada em: (1) medidores de esforços (SECCHI, 2010, p. 51) das políticas públicas para o esporte - legislação, financiamento, diretrizes e metas, planejamento, recursos humanos; (2) medidores de realização (SECCHI, 2010 p. 51) - estrutura, implementação, acompanhamento, avaliação, divulgação (de cada programa, projeto e ação), especificidade, produção do conhecimento e perenidade. Cada um desses indicadores deve conter uma relação de questões que servirão de fundamento para as análises qualitativas e quantitativas das pesquisas que serão desenvolvidas futuramente.

Em relação aos medidores de esforços, procurou-se compreender como se estruturam cada uma das categorias levantadas. O primeiro medidor de esforço trabalhado foi o relacionado ao aspecto legislativo, visto que tal elemento é algo primordial para uma compreensão da política pública, pois um adequado entendimento do marco regulatório mostra uma faceta importante dos programas, projetos e ações realizados por determinado governo.

Após o medidor legislativo, abordou-se o aspecto relativo ao financiamento, buscando visualizar como se constituem as formas de repasse monetário para as políticas públicas esportivas no Brasil. Os outros medidores de esforço selecionados foram diretrizes e metas, planejamento e recursos humanos. A compreensão de cada um desses itens se torna necessária para o entendimento global da política esportiva brasileira, fornecendo uma base segura para a compreensão dos medidores de realização.

Já os medidores de realização são necessários para uma compressão total do ciclo da política pública propriamente dita. Os medidores estrutura e implementação que cada política apresenta são categorias fundamentais para o funcionamento das políticas esportivas. A visualização de medidores como acompanhamento, avaliação e divulgação mostra como cada política se movimenta dentro do contexto social, contornando pontos relativos à sua efetividade.

A produção do conhecimento busca ver se tais ações governamentais se tornaram alvo de atenção de estudos acadêmicos mais sistematizados, mostrando o amadurecimento e/ou o não amadurecimento de cada programa, projeto e ação governamental. Por fim, a categoria perenidade busca visualizar se tais políticas públicas de esporte se cristalizam na sociedade de uma forma mais ampla e/ou se tornam medidas paliativas.

Para dar vazão a todos esses tópicos, dois instrumentos de pesquisa foram desenvolvidos de forma independente para coletar dados: (1) a definição de indicadores da política pública propriamente dita, conforme descrito acima; e (2) a elaboração de uma matriz analítica para cada política implementada pelos governos. Esses instrumentos de pesquisa foram amplamente discutidos para evitar dificuldades no momento de interpretação, bem como para aumentar a validade dos dados.

Em relação aos indicadores gerais da política, foram consultados diversos pesquisadores e os mesmos foram submetidos à 
validação. Esses indicadores posteriormente foram validados com base nos dados já coletados do programa federal "Bolsa-Atleta" (CORREA, 2013) e, em seguida, foram testados em outros programas, projetos e ações do governo federal. A padronização dos indicadores e da metodologia visa garantir referência de comparação entre as diferentes políticas públicas estabelecidas no país, no sentido de obter resultados mais amplos e robustos sobre as políticas públicas de esporte.

Já para a interpretação de cada programa, foi elaborado um questionário, dividido em questões fechadas e abertas. As perguntas abertas procuraram obter insights sobre cada ação. Já as perguntas fechadas foram adicionadas para garantir um grau de comparabilidade para os vários indicadores, permitindo a quantificação dos dados. As questões abertas também deram aos pesquisadores uma oportunidade para fornecer mais detalhes sobre possíveis critérios adicionais, que não foram incluídas nos questionários originais, mas que foram questões específicas de cada programa.

O grau de detalhes exigidos para preencher o questionário foi tal que a tarefa já era por si só uma investigação, pois os pesquisadores tiveram que procurar pesquisas nacionais e internacionais existentes e analisar as fontes e documentos da política pública do esporte no Brasil. Além disso, dependendo dos dados disponíveis e do conhecimento do investigador, entrevistas com membros dos demais órgãos do esporte no âmbito dos governos federal, estaduais e municipais deveriam ser realizadas para dar respostas a todos os pontos colocados no instrumento de pesquisa.

Os pesquisadores receberam orientações escritas detalhadas para ajudar com a conclusão dos instrumentos gerais e, para esclarecer possíveis ambiguidades, métodos foram discutidos durante uma reunião de revisão. Um fluxograma foi apresentado com mais instruções sobre a busca de fontes e dados. Os pesquisadores foram obrigados a fornecer detalhes abrangentes em suas respostas. Além disso, os pesquisadores receberam um exemplo dos indicadores para assegurar que as questões foram interpretadas corretamente.

Nesse sentido, a pesquisa serviu a dois propósitos: (1) reunir informações sobre os indicadores pré-definidos; e (2) para avaliar a qualidade do desenvolvimento de política pública de esporte no Brasil (SECCHI, 2010). Para finalizar os indicadores depois das adaptações, os mesmos foram novamente validados com os dados já catalogados do programa "Bolsa-Atleta" (CORRÊA, 2013).

Com isso, um protocolo de pesquisa foi elaborado visando aumentar a confiabilidade dos instrumentos, padronizar os procedimentos e principalmente para facilitar as comparações entre políticas públicas diferenciadas de esportes. Esse protocolo continha uma descrição minuciosa dos métodos propostos, definições dos grupos-alvo e as diretrizes para serem incluídos na pesquisa. Além disso, foi enfatizado que os questionários deveriam permanecer inalterados, sempre que possível, para garantir consistência da coleta de dados e comparação entre as amostras. Perguntas poderiam ser adicionadas à pesquisa pelos pesquisadores, contudo, jamais poderiam ser excluídas (SECCHI, 2010).

Tais procedimentos têm como objetivo os seguintes pontos: a) validar e refinar o modelo conceitual (especialmente para melhorar o conteúdo e validade 
de constructo); b) definir os indicadores e traduzi-los em questões de medição; c) supervisionar a objetividade da análise dos dados, a validade interna e confiabilidade; e, especialmente, d) garantir a comparabilidade dos dados.

\section{Construção da Matriz analítica de cada programa, projeto e ação governamental no campo esportivo}

A importância da construção da matriz analítica é de fundamental importância para aumentar a validade dos dados e permitir uma pesquisa empírica com dados altamente consistentes. Ao partir da premissa que só se quantifica o que sabe que existe, a etapa anterior foi de fundamental importância para estabelecer as bases e o terreno no qual a pesquisa deve ser construída e conduzida. Normalmente, costuma-se estabelecer o espaço específico da pesquisa quantitativa como aquele em que se busca contar e medir características dos fatos sociais. Enquanto que, por outro lado, os métodos qualitativos servem para classificar as características do objeto de análise. O problema, ou melhor, a principal limitação desse tipo de distinção está na impossibilidade de aplicá-la na prática da pesquisa científica.

Nesse sentido, a pesquisa quantitativa deve ser usada quando o que se quer medir no objeto pesquisado já é conhecido. Os métodos quantitativos são apropriados para estudar características do objeto de pesquisa que, sabe-se, existem, ao passo que os métodos qualitativos devem ser usados quando a pesquisa busca entender características do objeto que não se sabe se existem; porém, não há pesquisa social produtiva que utilize com exclusividade técnicas quantitativas ou qualitativas.

Sendo assim, trabalhar com dados é de fundamental importância para podermos transformá-los em informações/conhecimentos, compará-los com outros resultados e/ou, ainda, julgar sua adequação a alguma teoria. Conforme apontam Morettin e Bussab (2011), a essência da Ciência é a observação e seu objetivo básico é a denominada inferência. Esta, segundo os autores, é uma das partes da Estatística que tem por objetivo a coleta, redução, análise e modelagem dos dados, a partir do que, finalmente, faz-se a inferência para uma população da qual os dados (a amostra) foram obtidos. Um aspecto importante da modelagem dos dados é fazer previsões, a partir das quais se podem tomar decisões.

Por esses motivos, a criação de uma matriz analítica torna-se necessária para operacionalizar os pontos acima elencados, pois, somente com esta forma de procedimento, será possível realizar uma sistematização adequada das variáveis a serem analisadas. Para a formulação de uma matriz se adequar ao âmbito do qual se quer retirar os dados é de fundamental importância. Por isso, o questionário inicial é de basilar importância, pois o mesmo fornecerá as bases para a definição das variáveis que serão tratadas e analisadas. Na elaboração da matriz, duas perguntas devem sempre ser as balizadoras da operacionalização dos procedimentos: Qual informação deve ser coletada? Porque devem ser coletadas?

Cumprida essa etapa, uma padronização da matriz deve ser realizada e ela necessita ter duas características básicas: 1) a matriz deve conter um cabeçalho que apresente as variáveis estipuladas que deverão ser analisadas; e 2) o conteúdo das 
linhas desta matriz será preenchido pelos dados coletados. Tais dados devem ser sistematizados de acordo com um único padrão, qualquer modificação compromete a leitura e tratamento estatístico adequado.

O passo seguinte na construção desta matriz analítica foi a transposição dos dados de pesquisa coletados junto ao programa "Bolsa Atleta" de uma planilha de Excel para um banco de dados mais consistente. Para isso, foram contratados profissionais habilitados na área de tecnologia da informação, um banco de dados foi elaborado e um domínio on-line (http://praticaclinica.com.br/ie/) foi criado para que os pesquisadores pudessem catalogar os dados oriundos de suas pesquisas.

Trata-se de um sistema amplo que fornece a possibilidade de cadastrar todos os medidores estabelecidos na fase anterior da pesquisa. Como o sistema foi iniciado com os dados da pesquisa sobre o "Bolsa Atleta", priorizou-se a criação de um banco de dados que fornecesse todas as informações possíveis de cada modalidade esportiva presente no programa. Os dados coletados versavam desde os dados gerais sobre o atleta, passando pela quantidade de bolsas recebidas até chegar ao histórico competitivo do mesmo. Este sistema já foi ampliado para o programa "Atleta Pódio" e sua articulação com outros dois programas federais - "Centro de Iniciação ao Esporte" e "Jogos Escolares" - já se encontra em fase de implementação.

Este banco de dados possibilita a criação de um sistema altamente confiável do ponto de vista estatístico e que cruzado com o método de pesquisa proposto poderá fornecer elementos fundamentais para a avaliação das políticas públicas de esporte dentro de um padrão científico.

\section{CONSIDERAÇÕES FINAIS}

Este artigo teve como objetivo principal propor um método para medir e avaliar as políticas públicas de esporte no Brasil. Por isso, esforçou-se em abrir um diálogo sobre métodos para compreender as políticas de esporte no país. Uma das principais conclusões a que se chegou com o presente estudo é que as análises qualitativas, como os resultados quantitativos por si só, não são suficientes para avaliar a qualidade e/ou efetividade de uma política publica esportiva. Ao contrário dos experimentos de laboratório, os estudos sobre as políticas de esporte não são um sistema fechado. Estudos com este objeto necessitam tanto dos métodos qualitativos como de instrumentos quantitativos, para a realização de uma pesquisa profícua do ponto de vista acadêmico.

A presente proposta procurou desenvolver todo um modelo teórico que se utiliza de métodos de pesquisa mistos, ou seja, realiza um sistema de medição quantitativa como técnicas para comparar os nossos conhecimentos sobre o assunto sem deixar de ser amparado por um refinado modelo conceitual. Sendo assim, os resultados podem revelar uma série de critérios que não podem ser medidos e avaliados para o aprimoramento das políticas públicas de esporte no Brasil.

Os resultados oriundos dessas análises podem ser utilizados como um meio para medir a eficácia destes programas, projetos e ações. O significado desta proposta é a busca permanente por um conjunto confiável e válido de critérios com a capacidade de ser aplicado a diferentes casos, podendo contribuir tanto no desenvolvimento acadêmico/ científico como das ações políticas relativas ao esporte. 


\section{REFERÊNCIAS}

AMARAL, S. C. F. E PEREIRA, A. P. C. Reflexões sobre a produção em políticas públicas de Educação Física, esporte e lazer. Revista Brasileira de Ciências do Esporte, v. 31, n. 1. Campinas: Autores Associados, 2009.

BERGSGARD, N.A., et. al. Sport policy. A comparative analysis of stability and change. London: Elsevier, 2007.

BOURDIEU. P. Coisas ditas. São Paulo: Brasiliense, 1983a.

- Questões de sociologia. Rio de Janeiro: Marco Zero, 1983b.

O poder simbólico. Rio de Janeiro, Bertrand Brasil, 2009.

- Sobre o Estado: Cursos no Collège de France (1989-1992). São Paulo: Companhia das Letras, 2014.

BUGGEL, E. The development of Sport in the German Democratic Republic: 1950- 1985. Proceedings of the third International Seminar on Comparative Physical Education and Sport. Champaign, IL: Human Kinetics, 1986, p. 37-53.

CANAN, F. et. al. A configuração da Rede SETI Esportes: Discutindo e avaliando a partir da policy analys. Rev. Educ. Fís/ UEM, v. 25, n. 3, p. 391-403, 2014.

CHALIP, L. Policy analysis in sport management. Journal of Sport Management, 9, 1995, p.1-13.

COAKLEY, J. Using Social Research and Theory to Inform Public Policies that Integrate Leisure, Sport and Education. Texto apresentado no I Sem. Latino Americano de Políticas Públicas Integradas de Lazer, Esporte e Educação: Consolidando uma Rede de Produção do Conhecimento. Formação e Informação. Foz do Iguaçu, 2010.
CORRÊA, A. J. Financiamento do esporte olímpico brasileiro: mapeamento inicial do programa "Bolsa-Atleta". 2013. 86f. Monografia (Graduação em Educação Física) - Departamento de Educação Física. Universidade Federal do Paraná, Curitiba, 2013.

CRESWELL, J.W. E PLANO CLARK, V.L. Designing and conducing mixed methods research. London: Sage, 2007. . Pesquisa de métodos misto. Porto Alegre: Penso, 2013.

DACOSTA, L. E MIRAGAYA, A. Worldwide experiences and trends in sport for all. Oxford, UK: Meyer \& Meyer Sport, 2002.

DEUBel, A. N. R. Políticas Públicas: formulación, implementación e evolución. Bogotá, 2006

DIGEL, H., BURK, V.; FAHRNER, M. High-performnce sport. An international comparison. Edition Sports International, 9. Weilheim/Teck. Tubingen, DE: Bräuer, 2006.

DYE, T. R. Policy analysis. Alabama: University of Alabama Press, 1976.

FREY, K. Análise de políticas públicas: Algumas reflexões e suas implicações para a situação brasileiras. Cadernos de Pesquisa, $\mathrm{n}^{\circ} 18$, setembro. PPGSP/ UFSC. 2000.

HAAG, H. Triangulation: A strategy for upgrading comparative research methodology in sport science. In: WILCOX, R. (Ed.), Sport in the global village. Morgantown, WV: Fitness Information Technology, 1994, p. 501-507.

HÚNGARO, E. M. et. al. Balanço inicial da produção do GTT de políticas públicas do CBCE (1997-2005): avanços, ausências e perspectivas. 
In: HÚNGARO, E. M.; SOUSA, W. L. L. de (Org.). Cultura, educação, lazer e esporte: fundamentos, balanços e anotações críticas. Santo André: Alpharrabio, 2009, p. 93-124.

GODOY, L. O Sistema Nacional de Esporte no Brasil: revelações e possíveis delineamentos. 2013. 164 f. Tese (Doutorado em Educação Física) Universidade Federal do Paraná, Curitiba, 2013.

GREEN, M., E COLlins, S. Policy, politics and path dependency: Sport development in Australia and Finland. Sport Management Review, 11, 2008, p. 225-251.

GREEN, M.; OAKLEY, B. Elite sport development systems and playing to win: Uniformity and diversity in international approaches. Leisure Studies, 20, 2001, p. 247-267.

GREEN, M.; HOULIHAN, B. Elite sport development. Policy learning and political priorities. London: Routledge, 2005.

HAM, C.; HILL, M. The policy process in the modern capitalist state. London: Harvester Wheatsheaf, 1993.

HENRY, I.; AMARA, M.; AL-TAUQI, M. A typology of approaches to comparative analysis of sports policy. Journal of Sport Management, 19, 2005, p. 520-535.

LINHALES, M. A.; PEREIRA FILHO, J. R. Intervenção, conhecimento e mudança: a Educação Física, o esporte e o lazer nas políticas públicas. In: GOELLNER, S. V. Educação Física / Ciências do Esporte: intervenção e conhecimento. Campinas, SP: Autores Associados, 1999.

MANHÃES, E. D. Políticas de esportes no

Brasil. Rio de Janeiro: Graal, 1986.
MARTINES, I. C. As relações entre as Organizações Não Governamentais e o Governo do Paraná no Campo Esportivo. 212p. Dissertação (Mestrado em Educação Física). Departamento de Educação Física, Universidade Federal do Paraná, Curitiba, 2009.

MEZZADRI, F. M. Políticas Públicas para o Esporte e lazer: teorias e conceitos. In: Alexandre Motta \& Rodrigo Terra. (Org.). Esporte, Lazer e políticas públicas na região dos Lagos. Rio de Janeiro: iVentura, 2011, p. 89-102.

MORETTIN, P. A.; BUSSAB, W. O, Estatística Básica. São Paulo: Saraiva, 2011.

RIORDAN, J. Sport, politics and communism. Manchester, UK: Manchester University Press, 1991.

SEDLACEK, J. et. al. The influence of the political changes on the high performance sport organisation in Czechoslovakia. In: WILCOX, R. (Ed.). Sport in the global village. Morgantown, WV: Fitness Information Technology, 1994, p. 341-347. .

SOUZA, C. Políticas Públicas: uma revisão da literatura. Sociologias, Porto Alegre, ano $8, n^{\circ} 16$, jul/dez 2006, p. 20-45

Starepravo, F. A. Políticas Públicas de Esporte e Lazer no Brasil: Aproximações, intersecções, rupturas e distanciamentos entre os subcampos políticos/burocráticos e científico/ acadêmico. 2011. 422f. Tese (Doutorado em Educação Física) Programa de Pós-Graduação em Educação Física. Universidade Federal do Paraná, Curitiba, 2011. 
DEVELOPMENT OF A METHOD FOR RESEARCH IN SPORTS POLICY IN BRAZIL: an approach to method of mixed research

\begin{abstract}
This article presents a method of mixed research for the study of Sport public policy in Brazil. To reach the result, the text was divided into two phases. In the first, it presents a conceptual model for the qualitative analysis of Brazilian public policies directed to the sport. This phase will still be divided into two stages. The first is the construction of a theoretical framework for the interpretation of the study on public policies and the other one demonstrates a conceptual methodological contribution based on the sociology of Pierre Bourdieu. In the second phase it is developed an analysis of the quantitative part which aims to interpret the data that were collected in the empirical part of the research. Later, it will be possible to produce a model of assessment, monitoring, and, especially, the improvement of public policies for Brazilian sport.
\end{abstract}

Keywords: Sport Public Policies; State; Method of Mixed Research

\title{
DESARROLLO DE UN MÉTODO PARA LAS INVESTIGACIONES EN POLÍTICAS PÚBLICAS DE DEPORTE EN BRASIL: un abordaje de investigación mixta
}

\section{RESUMEN}

El artículo que se presenta expone um método de investigación mixta para el estudio de las políticas públicas de deporte en Brasil. Para atender a este fin, el texto se divide en dos etapas. En un primer momento se presenta un modelo conceptual para el análisis cualitativo de las polítcas públicas brasileñas dirigidas al deporte. Esta etapa aun se divide en otras dos. Una de ellas trata de la construcción de un referencial teórico para la interpretación del estudio sobre las políticas públicas y la otra demuestra un aporte conceptual metodológico basado en la sociología de Pierre Bourdieu. En la segunda etapa, se desarrolla un análisis de la parte cuantitativa que tiene por objetivo la interpretación de los datos recogidos en la parte empírica de la investigación. Después de estos pasos será posible, en una etapa posterior, producir un modelo de evaluación, monitorización y, principalmente, mejoras en las políticas públicas para el deporte brasileño.

Palabras Clave: Políticas Públicas de Deporte; Estado; Método de Investigación Mixta

Recebido em: dezembro/2014 Aprovado em: março/2015 